\title{
Solutions of Linear Equations
}

\author{
Karol Pąk \\ Institute of Computer Science \\ University of Białystok \\ Poland
}

\begin{abstract}
Summary. In this paper I present the Kronecker-Capelli theorem which states that a system of linear equations has a solution if and only if the rank of its coefficient matrix is equal to the rank of its augmented matrix.
\end{abstract}

MML identifier: MATRIX15, version: $\underline{7.8 .09 \quad 4.97 .1001}$

The terminology and notation used in this paper are introduced in the following papers: [9], [24], [1], [2], [10], [25], [6], [8], [7], [3], [23], [21], [13], [5], [11], [12], [26], [15], [27], [19], [16], [22], [20], [28], [4], [17], [14], and [18].

\section{Preliminaries}

For simplicity, we follow the rules: $x$ denotes a set, $i, j, k, l, m, n$ denote natural numbers, $K$ denotes a field, $N$ denotes a without zero finite subset of $\mathbb{N}, a, b$ denote elements of $K, A, B, B_{1}, B_{2}, X, X_{1}, X_{2}$ denote matrices over $K$, $A^{\prime}$ denotes a matrix over $K$ of dimension $m \times n, B^{\prime}$ denotes a matrix over $K$ of dimension $m \times k$, and $M$ denotes a square matrix over $K$ of dimension $n$.

We now state a number of propositions:

(1) If width $A=$ len $B$, then $(a \cdot A) \cdot B=a \cdot(A \cdot B)$.

(2) $\mathbf{1}_{K} \cdot A=A$ and $a \cdot(b \cdot A)=(a \cdot b) \cdot A$.

(3) Let $K$ be a non empty additive loop structure and $f, g, h, w$ be finite sequences of elements of $K$. If len $f=\operatorname{len} g$ and len $h=\operatorname{len} w$, then $f^{\frown}$ $h+g \frown w=(f+g) \frown(h+w)$.

(4) Let $K$ be a non empty multiplicative magma, $f, g$ be finite sequences of elements of $K$, and $a$ be an element of $K$. Then $a \cdot\left(f^{\frown} g\right)=(a \cdot f)^{\frown}(a \cdot g)$. 
(5) Let $f$ be a function and $p_{1}, p_{2}, f_{1}, f_{2}$ be finite sequences. If $\operatorname{rng} p_{1} \subseteq$ $\operatorname{dom} f$ and $\operatorname{rng} p_{2} \subseteq \operatorname{dom} f$ and $f_{1}=f \cdot p_{1}$ and $f_{2}=f \cdot p_{2}$, then $f \cdot\left(p_{1} \frown p_{2}\right)=$ $f_{1} \frown f_{2}$.

(6) Let $f$ be a finite sequence of elements of $\mathbb{N}$ and given $n$. Suppose $f$ is one-to-one and $\operatorname{rng} f \subseteq \operatorname{Seg} n$ and for all $i, j$ such that $i, j \in \operatorname{dom} f$ and $i<j$ holds $f(i)<f(j)$. Then Sgm rng $f=f$.

(7) Let $K$ be an Abelian add-associative right zeroed right complementable non empty additive loop structure, $p$ be a finite sequence of elements of $K$, and given $i, j$. Suppose $i, j \in \operatorname{dom} p$ and $i \neq j$ and for every $k$ such that $k \in \operatorname{dom} p$ and $k \neq i$ and $k \neq j$ holds $p(k)=0_{K}$. Then $\sum p=p_{i}+p_{j}$.

(8) If $i \in \operatorname{Seg} m$, then $(\operatorname{Sgm}(\operatorname{Seg}(n+m) \backslash \operatorname{Seg} n))(i)=n+i$.

(9) Let $D$ be a non empty set, $A$ be a matrix over $D$, and $B_{3}, B_{4}, C_{1}$, $C_{2}$ be without zero finite subsets of $\mathbb{N}$. Suppose $B_{3} \times B_{4} \subseteq$ the indices of $A$ and $C_{1} \times C_{2} \subseteq$ the indices of $A$. Let $B$ be a matrix over $D$ of dimension card $B_{3} \times$ card $B_{4}$ and $C$ be a matrix over $D$ of dimension card $C_{1} \times \operatorname{card} C_{2}$. Suppose that for all natural numbers $i, j, b_{1}, b_{2}, c_{1}$, $c_{2}$ such that $\langle i, j\rangle \in\left(B_{3} \times B_{4}\right) \cap\left(C_{1} \times C_{2}\right)$ and $b_{1}=\left(\operatorname{Sgm} B_{3}\right)^{-1}(i)$ and $b_{2}=\left(\operatorname{Sgm} B_{4}\right)^{-1}(j)$ and $c_{1}=\left(\operatorname{Sgm} C_{1}\right)^{-1}(i)$ and $c_{2}=\left(\operatorname{Sgm} C_{2}\right)^{-1}(j)$ holds $B_{b_{1}, b_{2}}=C_{c_{1}, c_{2}}$. Then there exists a matrix $M$ over $D$ of dimension len $A \times$ width $A$ such that $\operatorname{Segm}\left(M, B_{3}, B_{4}\right)=B$ and $\operatorname{Segm}\left(M, C_{1}, C_{2}\right)=C$ and for all $i, j$ such that $\langle i, j\rangle \in($ the indices of $M) \backslash\left(B_{3} \times B_{4} \cup C_{1} \times C_{2}\right)$ holds $M_{i, j}=A_{i, j}$.

(10) Let $P, Q, Q^{\prime}$ be without zero finite subsets of $\mathbb{N}$. Suppose $P \times Q^{\prime} \subseteq$ the indices of $A$. Let given $i, j$. Suppose $i \in \operatorname{dom} A \backslash P$ and $j \in \operatorname{Seg}$ width $A \backslash Q$ and $A_{i, j} \neq 0_{K}$ and $Q \subseteq Q^{\prime}$ and $\operatorname{Line}(A, i) \cdot \operatorname{Sgm} Q^{\prime}=\operatorname{card} Q^{\prime} \mapsto 0_{K}$. Then $\operatorname{rk}(A)>\operatorname{rk}(\operatorname{Segm}(A, P, Q))$.

(11) For every $N$ such that $N \subseteq \operatorname{dom} A$ and for every $i$ such that $i \in \operatorname{dom} A \backslash N$ holds $\operatorname{Line}(A, i)=$ width $A \mapsto 0_{K} \operatorname{holds} \operatorname{rk}(A)=$ $\operatorname{rk}(\operatorname{Segm}(A, N, \operatorname{Seg}$ width $A))$.

(12) For every $N$ such that $N \subseteq$ Seg width $A$ and for every $i$ such that $i \in$ Seg width $A \backslash N$ holds $A_{\square, i}=\operatorname{len} A \mapsto 0_{K}$ holds $\operatorname{rk}(A)=$ $\operatorname{rk}(\operatorname{Segm}(A, \operatorname{Seg} \operatorname{len} A, N))$.

(13) Let $V$ be a vector space over $K, U$ be a finite subset of $V, u, v$ be vectors of $V$, and given $a$. If $u, v \in U$, then $\operatorname{Lin}((U \backslash\{u\}) \cup\{u+a \cdot v\})$ is a subspace of $\operatorname{Lin}(U)$.

(14) Let $V$ be a vector space over $K, U$ be a finite subset of $V, u, v$ be vectors of $V$, and given $a$. Suppose $u, v \in U$ and if $u=v$, then $a \neq-\mathbf{1}_{K}$ or $u=0_{V}$. Then $\operatorname{Lin}((U \backslash\{u\}) \cup\{u+a \cdot v\})=\operatorname{Lin}(U)$. 


\section{Selected Properties of Joining Operation of two Matrices}

Let $D$ be a non empty set, let $n, m, k$ be natural numbers, let $A$ be a matrix over $D$ of dimension $n \times m$, and let $B$ be a matrix over $D$ of dimension $n \times k$. Then $A \frown B$ is a matrix over $D$ of dimension $n \times($ width $A+$ width $B)$.

We now state a number of propositions:

(15) Let $D$ be a non empty set, $A$ be a matrix over $D$ of dimension $n \times m$, $B$ be a matrix over $D$ of dimension $n \times k$, and given $i$. If $i \in \operatorname{Seg} n$, then $\operatorname{Line}(A \frown B, i)=\operatorname{Line}(A, i)^{\frown} \operatorname{Line}(B, i)$.

(16) Let $D$ be a non empty set, $A$ be a matrix over $D$ of dimension $n \times m$, $B$ be a matrix over $D$ of dimension $n \times k$, and given $i$. If $i \in \operatorname{Seg}$ width $A$, then $(A \frown B)_{\square, i}=A_{\square, i}$.

(17) Let $D$ be a non empty set, $A$ be a matrix over $D$ of dimension $n \times m$, $B$ be a matrix over $D$ of dimension $n \times k$, and given $i$. If $i \in \operatorname{Seg}$ width $B$, then $(A \frown B)_{\square \text {, width } A+i}=B_{\square, i}$.

(18) Let $D$ be a non empty set, $A$ be a matrix over $D$ of dimension $n$ $\times m, B$ be a matrix over $D$ of dimension $n \times k$, and $p_{3}, p_{4}$ be finite sequences of elements of $D$. If len $p_{3}=$ width $A$ and len $p_{4}=$ width $B$, then ReplaceLine $\left(A \frown B, i, p_{3} \frown p_{4}\right)=\left(\operatorname{ReplaceLine}\left(A, i, p_{3}\right)\right) \frown$ ReplaceLine $\left(B, i, p_{4}\right)$.

(19) Let $D$ be a non empty set, $A$ be a matrix over $D$ of dimension $n \times$ $m$, and $B$ be a matrix over $D$ of dimension $n \times k$. Then $\operatorname{Segm}(A-$ $B, \operatorname{Seg} n, \operatorname{Seg}$ width $A)=A$ and $\operatorname{Segm}(A \frown B, \operatorname{Seg} n, \operatorname{Seg}($ width $A+$ width $B) \backslash$ Seg width $A$ ) $=B$.

(20) For all matrices $A, B$ over $K$ such that len $A=\operatorname{len} B \operatorname{holds} \operatorname{rk}(A) \leq$ $\operatorname{rk}(A \frown B)$ and $\operatorname{rk}(B) \leq \operatorname{rk}(A \frown B)$.

(21) For all matrices $A, B$ over $K$ such that len $A=\operatorname{len} B$ and len $A=\operatorname{rk}(A)$ holds $\operatorname{rk}(A)=\operatorname{rk}(A \frown B)$.

(22) For all matrices $A, B$ over $K$ such that len $A=\operatorname{len} B$ and width $A=0$ holds $A \frown B=B$ and $B \frown A=B$.

(23) For all matrices $A, B$ over $K$ such that $B=0_{K}^{(\text {len } A) \times m} \operatorname{holds} \operatorname{rk}(A)=$ $\operatorname{rk}(A \frown B)$.

(24) Let $A, B$ be matrices over $K$. Suppose $\operatorname{rk}(A)=\operatorname{rk}(A \frown B)$ and len $A=$ len $B$. Let given $N$. Suppose $N \subseteq \operatorname{dom} A$ and for every $i$ such that $i \in N$ holds Line $(A, i)=$ width $A \mapsto 0_{K}$. Let given $i$. If $i \in N$, then $\operatorname{Line}(B, i)=$ width $B \mapsto 0_{K}$. 


\section{Basic Properties of two Transformations Which Transform Finite Sequences to Matrices}

For simplicity, we follow the rules: $D$ is a non empty set, $b_{3}$ is a finite sequence of elements of $D, b, f, g$ are finite sequences of elements of $K$, and $M_{1}$ is a matrix over $D$.

Let $D$ be a non empty set and let $b$ be a finite sequence of elements of $D$. The functor LineVec $2 \mathrm{Mx} b$ yielding a matrix over $D$ of dimension $1 \times \operatorname{len} b$ is defined by:

(Def. 1) LineVec2Mx $b=\langle b\rangle$.

The functor ColVec2Mx $b$ yielding a matrix over $D$ of dimension len $b \times 1$ is defined by:

(Def. 2) ColVec2Mx $b=\langle b\rangle^{\mathrm{T}}$.

One can prove the following propositions:

(25) $\quad M_{1}=\operatorname{LineVec} 2 \mathrm{Mx} b_{3}$ iff Line $\left(M_{1}, 1\right)=b_{3}$ and len $M_{1}=1$.

(26) If len $M_{1} \neq 0$ or len $b_{3} \neq 0$, then $M_{1}=\operatorname{ColVec} 2 \mathrm{Mx} b_{3}$ iff $\left(M_{1}\right)_{\square, 1}=b_{3}$ and width $M_{1}=1$.

(27) If len $f=\operatorname{len} g$, then LineVec2Mx $f+\operatorname{LineVec} 2 \mathrm{Mx} g=\operatorname{LineVec} 2 \mathrm{Mx}(f+$ $g)$.

(28) If len $f=\operatorname{len} g$, then ColVec2Mx $f+\operatorname{ColVec} 2 \mathrm{Mx} g=\operatorname{ColVec} 2 \mathrm{Mx}(f+g)$.

(29) $a \cdot \operatorname{LineVec} 2 \mathrm{Mx} f=\operatorname{LineVec} 2 \mathrm{Mx}(a \cdot f)$.

(30) $a \cdot \operatorname{ColVec} 2 \mathrm{Mx} f=\operatorname{ColVec} 2 \mathrm{Mx}(a \cdot f)$.

(31) $\operatorname{LineVec} 2 \mathrm{Mx}\left(k \mapsto 0_{K}\right)=0_{K}^{1 \times k}$.

(32) $\operatorname{ColVec} 2 \mathrm{Mx}\left(k \mapsto 0_{K}\right)=0_{K}^{k \times 1}$.

\section{Basis Properties of the Solution of Linear Equations}

Let us consider $K$ and let us consider $A, B$. The set of solutions of $A$ and $B$ is a set and is defined as follows:

(Def. 3) The set of solutions of $A$ and $B=\{X: \operatorname{len} X=$ width $A \wedge$ width $X=$ width $B \wedge A \cdot X=B\}$.

We now state a number of propositions:

(33) If the set of solutions of $A$ and $B$ is non empty, then len $A=\operatorname{len} B$.

(34) If $X \in$ the set of solutions of $A$ and $B$ and $i \in \operatorname{Seg}$ width $X$ and $X_{\square, i}=$ len $X \mapsto 0_{K}$, then $B \square, i=$ len $B \mapsto 0_{K}$.

(35) Suppose $X \in$ the set of solutions of $A$ and $B$. Then $a \cdot X \in$ the set of solutions of $A$ and $a \cdot B$ and $X \in$ the set of solutions of $a \cdot A$ and $a \cdot B$.

(36) If $a \neq 0_{K}$, then the set of solutions of $A$ and $B=$ the set of solutions of $a \cdot A$ and $a \cdot B$. 
(37) Suppose $X_{1} \in$ the set of solutions of $A$ and $B_{1}$ and $X_{2} \in$ the set of solutions of $A$ and $B_{2}$ and width $B_{1}=$ width $B_{2}$. Then $X_{1}+X_{2} \in$ the set of solutions of $A$ and $B_{1}+B_{2}$.

(38) If $X \in$ the set of solutions of $A^{\prime}$ and $B^{\prime}$, then $X \in$ the set of solutions of $\operatorname{RLine}\left(A^{\prime}, i, a \cdot \operatorname{Line}\left(A^{\prime}, i\right)\right)$ and $\operatorname{RLine}\left(B^{\prime}, i, a \cdot \operatorname{Line}\left(B^{\prime}, i\right)\right)$.

(39) Suppose $X \in$ the set of solutions of $A^{\prime}$ and $B^{\prime}$ and $j \in \operatorname{Seg} m$ and $i \neq j$. Then $X \in$ the set of solutions of $\operatorname{RLine}\left(A^{\prime}, i, \operatorname{Line}\left(A^{\prime}, i\right)+a \cdot \operatorname{Line}\left(A^{\prime}, j\right)\right)$ and $\operatorname{RLine}\left(B^{\prime}, i, \operatorname{Line}\left(B^{\prime}, i\right)+a \cdot \operatorname{Line}\left(B^{\prime}, j\right)\right)$.

(40) Suppose $j \in \operatorname{Seg} m$ and if $i=j$, then $a \neq-\mathbf{1}_{K}$. Then the set of solutions of $A^{\prime}$ and $B^{\prime}=$ the set of solutions of $\operatorname{RLine}\left(A^{\prime}, i, \operatorname{Line}\left(A^{\prime}, i\right)+a \cdot \operatorname{Line}\left(A^{\prime}, j\right)\right)$ and $\operatorname{RLine}\left(B^{\prime}, i, \operatorname{Line}\left(B^{\prime}, i\right)+a \cdot \operatorname{Line}\left(B^{\prime}, j\right)\right)$.

(41) If $X \in$ the set of solutions of $A$ and $B$ and $i \in \operatorname{dom} A$ and $\operatorname{Line}(A, i)=$ width $A \mapsto 0_{K}$, then Line $(B, i)=$ width $B \mapsto 0_{K}$.

(42) Let $n_{1}$ be an element of $\mathbb{N}^{n}$. Suppose $\operatorname{rng} n_{1} \subseteq \operatorname{dom} A$ and $n>$ 0 . Then the set of solutions of $A$ and $B \subseteq$ the set of solutions of $\operatorname{Segm}\left(A, n_{1}, \operatorname{Sgm} \operatorname{Seg}\right.$ width $\left.A\right)$ and $\operatorname{Segm}\left(B, n_{1}, \operatorname{Sgm} \operatorname{Seg}\right.$ width $\left.B\right)$.

(43) Let $n_{1}$ be an element of $\mathbb{N}^{n}$. Suppose $\operatorname{rng} n_{1} \subseteq \operatorname{dom} A=\operatorname{dom} B$ and $n>0$ and for every $i$ such that $i \in \operatorname{dom} A \backslash \operatorname{rng} n_{1}$ holds Line $(A, i)=$ width $A \mapsto 0_{K}$ and $\operatorname{Line}(B, i)=$ width $B \mapsto 0_{K}$. Then the set of solutions of $A$ and $B=$ the set of solutions of $\operatorname{Segm}\left(A, n_{1}, \operatorname{Sgm} \operatorname{Seg}\right.$ width $\left.A\right)$ and $\operatorname{Segm}\left(B, n_{1}, \operatorname{Sgm} \operatorname{Seg}\right.$ width $\left.B\right)$.

(44) Let given $N$. Suppose $N \subseteq \operatorname{dom} A$ and $N$ is non empty. Then the set of solutions of $A$ and $B \subseteq$ the set of solutions of $\operatorname{Segm}(A, N$, Seg width $A$ ) and $\operatorname{Segm}(B, N$, Seg width $B)$.

(45) Let given $N$. Suppose $N \subseteq \operatorname{dom} A$ and $N$ is non empty and $\operatorname{dom} A=$ $\operatorname{dom} B$ and for every $i$ such that $i \in \operatorname{dom} A \backslash N$ holds $\operatorname{Line}(A, i)=$ width $A \mapsto 0_{K}$ and $\operatorname{Line}(B, i)=$ width $B \mapsto 0_{K}$. Then the set of solutions of $A$ and $B=$ the set of solutions of $\operatorname{Segm}(A, N$, Seg width $A)$ and $\operatorname{Segm}(B, N, \operatorname{Seg}$ width $B)$.

(46) Suppose $i \in \operatorname{dom} A$ and len $A>1$. Then the set of solutions of $A$ and $B \subseteq$ the set of solutions of the deleting of $i$-row in $A$ and the deleting of $i$ -row in $B$.

(47) Let given $A, B, i$. Suppose $i \in \operatorname{dom} A$ and len $A>1$ and $\operatorname{Line}(A, i)=$ width $A \mapsto 0_{K}$ and $i \in \operatorname{dom} B$ and $\operatorname{Line}(B, i)=$ width $B \mapsto 0_{K}$. Then the set of solutions of $A$ and $B=$ the set of solutions of the deleting of $i$-row in $A$ and the deleting of $i$-row in $B$.

(48) Let $A$ be a matrix over $K$ of dimension $n \times m, B$ be a matrix over $K$ of dimension $n \times k$, and $P$ be a function from $\operatorname{Seg} n$ into $\operatorname{Seg} n$. Then

(i) the set of solutions of $A$ and $B \subseteq$ the set of solutions of $A \cdot P$ and $B \cdot P$, and 
(ii) if $P$ is one-to-one, then the set of solutions of $A$ and $B=$ the set of solutions of $A \cdot P$ and $B \cdot P$.

(49) Let $A$ be a matrix over $K$ of dimension $n \times m$ and given $N$. Suppose $\operatorname{card} N=n$ and $N \subseteq \operatorname{Seg} m$ and $\operatorname{Segm}(A, \operatorname{Seg} n, N)=I_{K}^{n \times n}$ and $n>0$. Then there exists a matrix $M_{2}$ over $K$ of dimension $m-^{\prime} n \times m$ such that

(i) $\operatorname{Segm}\left(M_{2}, \operatorname{Seg}\left(m-^{\prime} n\right), \operatorname{Seg} m \backslash N\right)=I_{K}^{\left(m-^{\prime} n\right) \times\left(m-^{\prime} n\right)}$,

(ii) $\operatorname{Segm}\left(M_{2}, \operatorname{Seg}\left(m-{ }^{\prime} n\right), N\right)=-(\operatorname{Segm}(A, \operatorname{Seg} n, \operatorname{Seg} m \backslash N))^{\mathrm{T}}$, and

(iii) for every $l$ and for every matrix $M$ over $K$ of dimension $m \times l$ such that for every $i$ such that $i \in \operatorname{Seg} l$ holds there exists $j$ such that $j \in \operatorname{Seg}\left(m-{ }^{\prime} n\right)$ and $M_{\square, i}=\operatorname{Line}\left(M_{2}, j\right)$ or $M_{\square, i}=m \mapsto 0_{K}$ holds $M \in$ the set of solutions of $A$ and $0_{K}^{n \times l}$.

(50) Let $A$ be a matrix over $K$ of dimension $n \times m, B$ be a matrix over $K$ of dimension $n \times l$, and given $N$. Suppose card $N=n$ and $N \subseteq \operatorname{Seg} m$ and $n>0$ and $\operatorname{Segm}(A, \operatorname{Seg} n, N)=I_{K}^{n \times n}$. Then there exists a matrix $X$ over $K$ of dimension $m \times l$ such that $\operatorname{Segm}(X, \operatorname{Seg} m \backslash N, \operatorname{Seg} l)=0_{K}^{\left(m-{ }^{\prime} n\right) \times l}$ and $\operatorname{Segm}(X, N, \operatorname{Seg} l)=B$ and $X \in$ the set of solutions of $A$ and $B$.

(51) Let $A$ be a matrix over $K$ of dimension $0 \times n$ and $B$ be a matrix over $K$ of dimension $0 \times m$. Then the set of solutions of $A$ and $B=\{\emptyset\}$.

(52) For every matrix $B$ over $K$ such that the set of solutions of $0_{K}^{n \times k}$ and $B$ is non empty holds $B=0_{K}^{n \times(\text { width } B)}$.

(53) Let $A$ be a matrix over $K$ of dimension $n \times k$ and $B$ be a matrix over $K$ of dimension $n \times m$. Suppose $n>0$. Suppose $x \in$ the set of solutions of $A$ and $B$. Then $x$ is a matrix over $K$ of dimension $k \times m$.

(54) Suppose $n>0$ and $k>0$. Then the set of solutions of $0_{K}^{n \times k}$ and $0_{K}^{n \times m}=$ $\{X: X$ ranges over matrices over $K$ of dimension $k \times m\}$.

(55) If $n>0$ and the set of solutions of $0_{K}^{n \times 0}$ and $0_{K}^{n \times m}$ is non empty, then $m=0$.

(56) The set of solutions of $0_{K}^{n \times 0}$ and $0_{K}^{n \times 0}=\{\emptyset\}$.

\section{Gaussian Eliminations}

In this article we present several logical schemes. The scheme GAUSS1 deals with a field $\mathcal{A}$, natural numbers $\mathcal{B}, \mathcal{C}, \mathcal{D}$, a matrix $\mathcal{E}$ over $\mathcal{A}$ of dimension $\mathcal{B} \times$ $\mathcal{C}$, a matrix $\mathcal{F}$ over $\mathcal{A}$ of dimension $\mathcal{B} \times \mathcal{D}$, a 4 -ary functor $\mathcal{F}$ yielding a matrix over $\mathcal{A}$ of dimension $\mathcal{B} \times \mathcal{D}$, and a binary predicate $\mathcal{P}$, and states that:

There exists a matrix $A^{\prime}$ over $\mathcal{A}$ of dimension $\mathcal{B} \times \mathcal{C}$ and there exists a matrix $B^{\prime}$ over $\mathcal{A}$ of dimension $\mathcal{B} \times \mathcal{D}$ and there exists a without zero finite subset $N$ of $\mathbb{N}$ such that

$N \subseteq \operatorname{Seg} \mathcal{C}$ and $\operatorname{rk}(\mathcal{E})=\operatorname{rk}\left(A^{\prime}\right)$ and $\operatorname{rk}(\mathcal{E})=\operatorname{card} N$ and $\mathcal{P}\left[A^{\prime}, B^{\prime}\right]$ and $\operatorname{Segm}\left(A^{\prime}, \operatorname{Seg} \operatorname{card} N, N\right)$ is diagonal and for every $i$ 
such that $i \in \operatorname{Seg} \operatorname{card} N$ holds $A_{i,(\operatorname{Sgm} N)_{i}}^{\prime} \neq 0_{\mathcal{A}}$ and for every $i$ such that $i \in \operatorname{dom} A^{\prime}$ and $i>\operatorname{card} N$ holds Line $\left(A^{\prime}, i\right)=\mathcal{C} \mapsto 0_{\mathcal{A}}$ and for all $i, j$ such that $i \in \operatorname{Seg} \operatorname{card} N$ and $j \in \operatorname{Seg}$ width $A^{\prime}$ and $j<(\operatorname{Sgm} N)(i)$ holds $A_{i, j}^{\prime}=0_{\mathcal{A}}$

provided the parameters meet the following requirements:

- $\mathcal{P}[\mathcal{E}, \mathcal{F}]$, and

- Let $A^{\prime}$ be a matrix over $\mathcal{A}$ of dimension $\mathcal{B} \times \mathcal{C}$ and $B^{\prime}$ be a matrix over $\mathcal{A}$ of dimension $\mathcal{B} \times \mathcal{D}$. Suppose $\mathcal{P}\left[A^{\prime}, B^{\prime}\right]$. Let given $i, j$. Suppose $i \neq j$ and $j \in \operatorname{dom} A^{\prime}$. Let $a$ be an element of $\mathcal{A}$. Then $\mathcal{P}\left[\operatorname{RLine}\left(A^{\prime}, i, \operatorname{Line}\left(A^{\prime}, i\right)+a \cdot \operatorname{Line}\left(A^{\prime}, j\right)\right), \mathcal{F}\left(B^{\prime}, i, j, a\right)\right]$.

The scheme GAUSS2 deals with a field $\mathcal{A}$, natural numbers $\mathcal{B}, \mathcal{C}, \mathcal{D}$, a matrix $\mathcal{E}$ over $\mathcal{A}$ of dimension $\mathcal{B} \times \mathcal{C}$, a matrix $\mathcal{F}$ over $\mathcal{A}$ of dimension $\mathcal{B} \times \mathcal{D}$, a 4-ary functor $\mathcal{F}$ yielding a matrix over $\mathcal{A}$ of dimension $\mathcal{B} \times \mathcal{D}$, and a binary predicate $\mathcal{P}$, and states that:

There exists a matrix $A^{\prime}$ over $\mathcal{A}$ of dimension $\mathcal{B} \times \mathcal{C}$ and there exists a matrix $B^{\prime}$ over $\mathcal{A}$ of dimension $\mathcal{B} \times \mathcal{D}$ and there exists a without zero finite subset $N$ of $\mathbb{N}$ such that

$N \subseteq \operatorname{Seg} \mathcal{C}$ and $\operatorname{rk}(\mathcal{E})=\operatorname{rk}\left(A^{\prime}\right)$ and $\operatorname{rk}(\mathcal{E})=\operatorname{card} N$ and $\mathcal{P}\left[A^{\prime}, B^{\prime}\right]$ and $\operatorname{Segm}\left(A^{\prime}, \operatorname{Seg} \operatorname{card} N, N\right)=I_{\mathcal{A}}^{\operatorname{card} N \times \operatorname{card} N}$ and for every $i$ such that $i \in \operatorname{dom} A^{\prime}$ and $i>\operatorname{card} N$ holds Line $\left(A^{\prime}, i\right)=$ $\mathcal{C} \mapsto 0_{\mathcal{A}}$ and for all $i, j$ such that $i \in \operatorname{Seg} \operatorname{card} N$ and $j \in$ Seg width $A^{\prime}$ and $j<(\operatorname{Sgm} N)(i)$ holds $A_{i, j}^{\prime}=0_{\mathcal{A}}$

provided the parameters satisfy the following conditions:

- $\mathcal{P}[\mathcal{E}, \mathcal{F}]$, and

- Let $A^{\prime}$ be a matrix over $\mathcal{A}$ of dimension $\mathcal{B} \times \mathcal{C}$ and $B^{\prime}$ be a matrix over $\mathcal{A}$ of dimension $\mathcal{B} \times \mathcal{D}$. Suppose $\mathcal{P}\left[A^{\prime}, B^{\prime}\right]$. Let $a$ be an element of $\mathcal{A}$ and given $i, j$. If $j \in \operatorname{dom} A^{\prime}$ and if $i=j$, then $a \neq-\mathbf{1}_{\mathcal{A}}$, then $\mathcal{P}\left[\operatorname{RLine}\left(A^{\prime}, i, \operatorname{Line}\left(A^{\prime}, i\right)+a \cdot \operatorname{Line}\left(A^{\prime}, j\right)\right), \mathcal{F}\left(B^{\prime}, i, j, a\right)\right]$.

\section{The Main Theorem}

We now state the proposition

(57) Let $A, B$ be matrices over $K$. Suppose len $A=\operatorname{len} B$ and if width $A=0$, then width $B=0$. Then $\operatorname{rk}(A)=\operatorname{rk}(A \frown B)$ if and only if the set of solutions of $A$ and $B$ is non empty.

\section{Space of Solutions of Linear Equations}

Let us consider $K$, let $A$ be a matrix over $K$, and let $b$ be a finite sequence of elements of $K$. The set of solutions of $A$ and $b$ is defined by: 
(Def. 4) The set of solutions of $A$ and $b=\{f:$ ColVec2Mx $f \in$ the set of solutions of $A$ and ColVec2Mx $b\}$.

We now state two propositions:

(58) For every $x$ such that $x \in$ the set of solutions of $A$ and ColVec2Mx $b$ there exists $f$ such that $x=\operatorname{ColVec} 2 \mathrm{Mx} f$ and len $f=$ width $A$.

(59) For every $f$ such that ColVec2Mx $f \in$ the set of solutions of $A$ and ColVec2Mx $b$ holds len $f=$ width $A$.

Let us consider $K$, let $A$ be a matrix over $K$, and let $b$ be a finite sequence of elements of $K$. Then the set of solutions of $A$ and $b$ is a subset of the width $A$ dimension vector space over $K$.

Let us consider $K$, let $A$ be a matrix over $K$, and let $k$ be an element of $\mathbb{N}$. Note that the set of solutions of $A$ and $k \mapsto 0_{K}$ is linearly closed.

We now state two propositions:

(60) If the set of solutions of $A$ and $b$ is non empty and width $A=0$, then len $A=0$.

(61) If width $A \neq 0$ or len $A=0$, then the set of solutions of $A$ and len $A \mapsto 0_{K}$ is non empty.

Let us consider $K$ and let $A$ be a matrix over $K$. Let us assume that if width $A=0$, then len $A=0$. The space of solutions of $A$ is a strict subspace of the width $A$-dimension vector space over $K$ and is defined by:

(Def. 5) The carrier of the space of solutions of $A=$ the set of solutions of $A$ and len $A \mapsto 0_{K}$.

The following propositions are true:

(62) Let $A$ be a matrix over $K$ and $b$ be a finite sequence of elements of $K$. Suppose the set of solutions of $A$ and $b$ is non empty. Then the set of solutions of $A$ and $b$ is a coset of the space of solutions of $A$.

(63) Let given $A$. Suppose if width $A=0$, then $\operatorname{len} A=0$ and $\operatorname{rk}(A)=0$. Then the space of solutions of $A=$ the width $A$-dimension vector space over $K$.

(64) For every $A$ such that the space of solutions of $A=$ the width $A$ dimension vector space over $K \operatorname{holds} \operatorname{rk}(A)=0$.

(65) Let given $i, j$. Suppose $j \in \operatorname{Seg} m$ and $n>0$ and if $i=j$, then $a \neq-\mathbf{1}_{K}$. Then the space of solutions of $A^{\prime}=$ the space of solutions of $\operatorname{RLine}\left(A^{\prime}, i, \operatorname{Line}\left(A^{\prime}, i\right)+a \cdot \operatorname{Line}\left(A^{\prime}, j\right)\right)$.

(66) Let given $N$. Suppose $N \subseteq \operatorname{dom} A$ and $N$ is non empty and width $A>0$ and for every $i$ such that $i \in \operatorname{dom} A \backslash N$ holds $\operatorname{Line}(A, i)=$ width $A \mapsto$ $0_{K}$. Then the space of solutions of $A=$ the space of solutions of $\operatorname{Segm}(A, N, \operatorname{Seg}$ width $A)$.

(67) Let $A$ be a matrix over $K$ of dimension $n \times m$ and given $N$. Suppose $\operatorname{card} N=n$ and $N \subseteq \operatorname{Seg} m$ and $\operatorname{Segm}(A, \operatorname{Seg} n, N)=I_{K}^{n \times n}$ and $n>0$ 
and $m-{ }^{\prime} n>0$. Then there exists a matrix $M_{2}$ over $K$ of dimension $m-^{\prime} n \times m$ such that $\operatorname{Segm}\left(M_{2}, \operatorname{Seg}\left(m-^{\prime} n\right), \operatorname{Seg} m \backslash N\right)=I_{K}^{\left(m-^{\prime} n\right) \times\left(m-^{\prime} n\right)}$ and $\operatorname{Segm}\left(M_{2}, \operatorname{Seg}\left(m-^{\prime} n\right), N\right)=-(\operatorname{Segm}(A, \operatorname{Seg} n, \operatorname{Seg} m \backslash N))^{\mathrm{T}}$ and $\operatorname{Lin}\left(\right.$ lines $\left.\left(M_{2}\right)\right)=$ the space of solutions of $A$.

(68) For every $A$ such that if width $A=0$, then len $A=0$ holds $\operatorname{dim}$ (the space of solutions of $A)=$ width $A-\operatorname{rk}(A)$.

(69) Let $M$ be a matrix over $K$ of dimension $n \times m$ and given $i, j, a$. Suppose $M$ is without repeated line and $j \in \operatorname{dom} M$ and if $i=j$, then $a \neq-\mathbf{1}_{K}$. Then $\operatorname{Lin}(\operatorname{lines}(M))=\operatorname{Lin}(\operatorname{lines}(\operatorname{RLine}(M, i, \operatorname{Line}(M, i)+a \cdot \operatorname{Line}(M, j))))$.

(70) Let $W$ be a subspace of the $m$-dimension vector space over $K$. Then there exists a matrix $A$ over $K$ of dimension $\operatorname{dim}(W) \times m$ and there exists a without zero finite subset $N$ of $\mathbb{N}$ such that $N \subseteq \operatorname{Seg} m$ and $\operatorname{dim}(W)=\operatorname{card} N$ and $\operatorname{Segm}(A, \operatorname{Seg} \operatorname{dim}(W), N)=I_{K}^{\operatorname{dim}(W) \times \operatorname{dim}(W)}$ and $\operatorname{rk}(A)=\operatorname{dim}(W)$ and lines $(A)$ is a basis of $W$.

(71) Let $W$ be a strict subspace of the $m$-dimension vector space over $K$. Suppose $\operatorname{dim}(W)<m$. Then there exists a matrix $A$ over $K$ of dimension $m-{ }^{\prime} \operatorname{dim}(W) \times m$ and there exists a without zero finite subset $N$ of $\mathbb{N}$ such that $\operatorname{card} N=m-^{\prime} \operatorname{dim}(W)$ and $N \subseteq \operatorname{Seg} m$ and $\operatorname{Segm}\left(A, \operatorname{Seg}\left(m-{ }^{\prime}\right.\right.$ $\operatorname{dim}(W)), N)=I_{K}^{\left(m-{ }^{\prime} \operatorname{dim}(W)\right) \times\left(m-{ }^{\prime} \operatorname{dim}(W)\right)}$ and $W=$ the space of solutions of $A$.

(72) Let $A, B$ be matrices over $K$. Suppose width $A=\operatorname{len} B$ and if width $A=$ 0 , then len $A=0$ and if width $B=0$, then len $B=0$. Then the space of solutions of $B$ is a subspace of the space of solutions of $A \cdot B$.

(73) For all matrices $A, B$ over $K$ such that width $A=\operatorname{len} B$ holds $\operatorname{rk}(A \cdot B) \leq$ $\operatorname{rk}(A)$ and $\operatorname{rk}(A \cdot B) \leq \operatorname{rk}(B)$.

(74) Let $A$ be a matrix over $K$ of dimension $n \times n$ and $B$ be a matrix over $K$. Suppose Det $A \neq 0_{K}$ and width $A=\operatorname{len} B$ and if width $B=0$, then len $B=0$. Then the space of solutions of $B=$ the space of solutions of $A \cdot B$.

(75) Let $A$ be a matrix over $K$ of dimension $n \times n$ and $B$ be a matrix over $K$. If width $A=\operatorname{len} B$ and $\operatorname{Det} A \neq 0_{K}$, then $\operatorname{rk}(A \cdot B)=\operatorname{rk}(B)$.

(76) Let $A$ be a matrix over $K$ of dimension $n \times n$ and $B$ be a matrix over $K$. If len $A=$ width $B$ and $\operatorname{Det} A \neq 0_{K}$, then $\operatorname{rk}(B \cdot A)=\operatorname{rk}(B)$.

\section{REFERENCES}

[1] Grzegorz Bancerek. Cardinal numbers. Formalized Mathematics, 1(2):377-382, 1990.

[2] Grzegorz Bancerek. The fundamental properties of natural numbers. Formalized Mathematics, 1(1):41-46, 1990.

[3] Grzegorz Bancerek and Krzysztof Hryniewiecki. Segments of natural numbers and finite sequences. Formalized Mathematics, 1(1):107-114, 1990.

[4] Czesław Byliński. Binary operations applied to finite sequences. Formalized Mathematics, 1(4):643-649, 1990. 
[5] Czesław Byliński. Finite sequences and tuples of elements of a non-empty sets. Formalized Mathematics, 1(3):529-536, 1990.

[6] Czesław Byliński. Functions and their basic properties. Formalized Mathematics, 1(1):5565, 1990.

[7] Czesław Byliński. Functions from a set to a set. Formalized Mathematics, 1(1):153-164, 1990.

[8] Czesław Byliński. Partial functions. Formalized Mathematics, 1(2):357-367, 1990.

[9] Czesław Byliński. Some basic properties of sets. Formalized Mathematics, 1(1):47-53, 1990.

[10] Agata Darmochwał. Finite sets. Formalized Mathematics, 1(1):165-167, 1990.

[11] Katarzyna Jankowska. Matrices. Abelian group of matrices. Formalized Mathematics, $2(4): 475-480,1991$.

[12] Katarzyna Jankowska. Transpose matrices and groups of permutations. Formalized Mathematics, 2(5):711-717, 1991.

[13] Eugeniusz Kusak, Wojciech Leończuk, and Michał Muzalewski. Abelian groups, fields and vector spaces. Formalized Mathematics, 1(2):335-342, 1990.

[14] Robert Milewski. Associated matrix of linear map. Formalized Mathematics, 5(3):339$345,1996$.

[15] Takaya Nishiyama and Yasuho Mizuhara. Binary arithmetics. Formalized Mathematics, 4(1):83-86, 1993.

[16] Karol Pạk. Basic properties of determinants of square matrices over a field. Formalized Mathematics, 15(1):17-25, 2007.

[17] Karol Pạk. Basic properties of the rank of matrices over a field. Formalized Mathematics, 15(4):199-211, 2007.

[18] Karol Pạk and Andrzej Trybulec. Laplace expansion. Formalized Mathematics, 15(3):143$150,2007$.

[19] Andrzej Trybulec. Domains and their Cartesian products. Formalized Mathematics, 1(1):115-122, 1990.

[20] Wojciech A. Trybulec. Basis of vector space. Formalized Mathematics, 1(5):883-885, 1990.

[21] Wojciech A. Trybulec. Groups. Formalized Mathematics, 1(5):821-827, 1990.

[22] Wojciech A. Trybulec. Subspaces and cosets of subspaces in vector space. Formalized Mathematics, 1(5):865-870, 1990.

[23] Wojciech A. Trybulec. Vectors in real linear space. Formalized Mathematics, 1(2):291-296, 1990.

[24] Zinaida Trybulec. Properties of subsets. Formalized Mathematics, 1(1):67-71, 1990.

[25] Edmund Woronowicz. Relations and their basic properties. Formalized Mathematics, 1(1):73-83, 1990.

[26] Katarzyna Zawadzka. The sum and product of finite sequences of elements of a field. Formalized Mathematics, 3(2):205-211, 1992.

[27] Katarzyna Zawadzka. The product and the determinant of matrices with entries in a field. Formalized Mathematics, 4(1):1-8, 1993.

[28] Mariusz Żynel. The Steinitz theorem and the dimension of a vector space. Formalized Mathematics, 5(3):423-428, 1996. 